\title{
The effect of contrast agents on left ventricular parameters calculated by a threshold-based software module: does it truly matter?
}

\author{
Andrea Szücs ${ }^{1}$. Anna Réka Kiss ${ }^{1}$. Ferenc Imre Suhai ${ }^{1}$ - Attila Tóth ${ }^{1}$ • Zsófia Gregor ${ }^{1}$ - Márton Horváth ${ }^{1}$. \\ Csilla Czimbalmos ${ }^{1} \cdot$ Ibolya Csécs $^{1} \cdot$ Zsófia Dohy $^{1} \cdot$ Liliána Erzsébet Szabó $^{1} \cdot$ Béla Merkely $^{1} \cdot$ Hajnalka Vágó $^{1}$
}

Received: 18 December 2018 / Accepted: 23 March 2019 / Published online: 29 April 2019

(c) The Author(s) 2019

\begin{abstract}
The acquisition of short-axis (SA) cine magnetic resonance (MR) images after the administration of contrast agent (CA) is a common, time-saving technique, but a decreased difference in the blood-myocardium contrast on these steady-state free precession (SSFP) cine scans could change the calculated parameters when using threshold-based papillary and trabecular muscle (PTM) quantification. We studied the effect of CA on the parameters calculated from pre- and post-CA SA cine images in noncompaction cardiomyopathy (NC-CMP) and healthy (H) participants using a threshold-based module. A total of 39 individuals (20 patients and 19 healthy) were included prospectively in this study. After the pre-CA SA images were acquired, i.v. gadobutrol (GA) or gadobenate dimeglumine (GD) (GA vs. GD: NC-CMP $=12$ vs. 8; $\mathrm{C}=12$ vs. 7) was administered, and SA scans were repeated after two minutes. A threshold-based PTM software was used for postprocessing. Pre-CA and postCA SA images were analyzed, and the parameters were compared in both the NC-CMP and H groups. The left ventricular volumes were significantly larger, while the left ventricular myocardial (LVmass) and trabecular mass (LVtrab) values were significantly smaller on the post-CA scans (NC-CMP: pre-CA vs. post-CA, EDV: $74.0 \pm 13.6 \mathrm{vs.} 81.1 \pm 16.3 \mathrm{ml} / \mathrm{m}^{2}$, ESV: $25.3 \pm 7.3$ vs. $30.1 \pm 11.2 \mathrm{ml} / \mathrm{m}^{2}$, LVmass-ED: $82.5 \pm 17.5$ vs. $75.7 \pm 15.9 \mathrm{~g} / \mathrm{m}^{2}$, LVtrab-ED: $25.0 \pm 6.6$ vs. $18.9 \pm 4.7 \mathrm{~g} / \mathrm{m}^{2}$; Healthy: preCA vs. post-CA, EDV: $69.7 \pm 11.9$ vs. $72.2 \pm 10.7 \mathrm{ml} / \mathrm{m}^{2}$, ESV: $22.6 \pm 5.7$ vs. $23.9 \pm 6.3 \mathrm{ml} / \mathrm{m}^{2}$, LVmass-ED: $71.3 \pm 13.6$ vs. $68.7 \pm 13.9 \mathrm{~g} / \mathrm{m}^{2}$, LVtrab-ED: $19.4 \pm 2.6$ vs. $\left.16.2 \pm 3.0 \mathrm{~g} / \mathrm{m}^{2} ; \mathrm{p}<0.05\right)$. The decreased blood-myocardium contrast difference on post-CA SSFP SA cine images leads to altered cardiac parameters when using threshold-based software for evaluation.
\end{abstract}

Keywords Cardiac magnetic resonance $\cdot$ Left ventricular noncompaction $\cdot$ Contrast agent $\cdot$ Threshold-based trabecular quantification

\section{Introduction}

Postprocessing software has been developing continuously since cardiac magnetic resonance (CMR) imaging was first performed; thus, the currently available techniques are more user-friendly and are able to provide gold-standard data on

Andrea Szúcs and Anna Réka Kiss have contributed equally to this work.

Andrea Szúcs

szucsand@gmail.com

Anna Réka Kiss

annarekak@gmail.com

1 Heart and Vascular Center, Semmelweis University of Budapest, Budapest, Hungary cardiac function and mass [1]. One of the newest achievements of this technical development is threshold-based papillary and trabeculated muscle (PTM) quantification. Based on the different signal intensities of blood and myocardial tissue, these algorithms differentiate small endocardial trabeculae and papillary muscles from the blood pool without endocardial contours, resulting in more accurate data concerning volumetric parameters and myocardial mass $[2$, $3]$. These algorithms can play a role in postprocessing in patients with myocardial disorders with hypertrabeculation, as inclusion or exclusion of excessive endocardial trabeculae during traditional contouring can significantly change the measured volumes and mass $[4,5]$. In addition to the accuracy of the calculated data, the benefits of this approach include the ability to use the technique retrospectively without extra CMR images; the reproducibility of this approach 
is also excellent and independent of the experience of the observers [6].

Noncompaction cardiomyopathy (NC-CMP) is a rare disorder caused by the failure of myocardial compaction during embryogenesis and results in excessive trabeculation, mainly in the apical part of the left ventricle. The clinical significance of this disease is controversial; in many cases, left ventricle noncompaction ends in dilated cardiomyopathy and heart failure, but patients can also remain symptomless with good left ventricular ejection fraction values [7].

NC-CMP has been diagnosed at a higher rate in recent years due to the wider availability of CMR. To shorten the scan time, SSFP short-axis (SA) cine images are often acquired after contrast agent (CA) administration, which is a global practice and a recommended technique for CMR examinations [8-10]. Endocardial trabeculation is harder to visualize on these scans because the $\mathrm{CA}$ alters the signal intensity of the blood pool and the myocardium on SSFP images, decreasing the difference between them. Therefore, the calculated volumetric data and the myocardial mass can

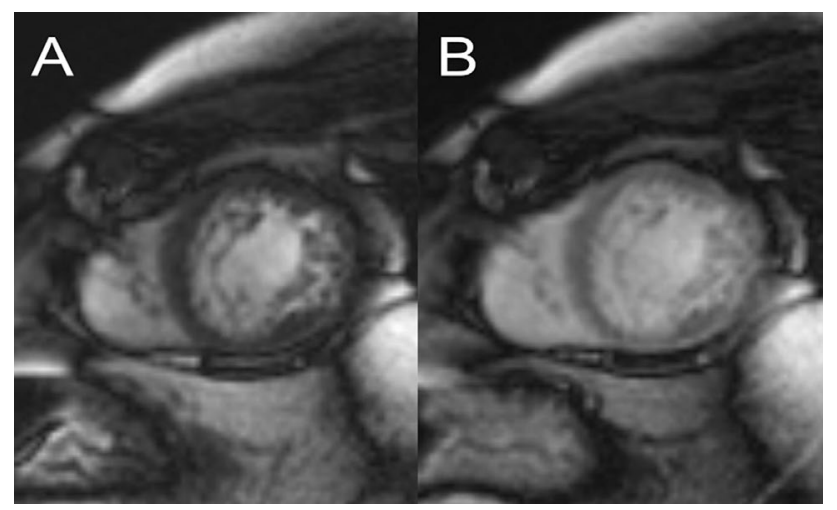

Fig. 1 Short axis images collected before (a) and after (b) injection of contrast agent be altered (Fig. 1). Moreover, the significance of this effect on the precision of another type of CMR analytical software, namely, feature tracking, has been reported in the literature [11].

Because limited data are available regarding the effect of CAs on postprocessing evaluation, we studied quantitative differences. The aim of our study was to quantify the effect of CA on calculated parameters using postprocessing software capable of threshold-based PTM quantification. First, pre- and postcontrast SSFP SA cine images of patients with left ventricular hypertrabeculation that were analyzed with this software were compared. Furthermore, we tested whether the loss of contrast difference between the blood and the myocardium caused alterations in the calculated parameters of a healthy normal population or whether this change plays a role only in patients with hypertrabeculation. Third, different types of gadolinium-based CAs show different relaxivity effects and slightly different kinetics; thus, we studied the effects of different contrast agents.

\section{Materials and methods}

\section{Patient characteristics}

Twenty NC-CMP patients with good left ventricular ejection fraction values and without any additional cardiac abnormalities or cardiovascular diseases and 19 healthy individuals were prospectively enrolled in 2016-2017. The exclusion criteria were the presence of congenital heart disease, ischemic heart disease, other cardiomyopathies or myocarditis in the patient's history. The baseline parameters of NC-CMP patients and healthy individuals are reported in Table 1. All procedures performed in this study were in accordance with the ethical standards of the institutional and national research committee and with the 1964 Helsinki
Table 1 Baseline characteristics of the noncompaction cardiomyopathy and healthy study groups

\begin{tabular}{llll}
\hline & NC-CMP $\mathrm{n}=20$ & Healthy $\mathrm{n}=19$ & $\mathrm{p}$ \\
\hline Gadobutrol $(\mathrm{n})$ & 12 & 12 & \\
Gadobenate dimenglumine $(\mathrm{n})$ & 8 & 7 & \\
Age $($ years $)$ & $41.7 \pm 16.3$ & $37.9 \pm 16.6$ & 0.4736 \\
EF $(\%)$ & $66.1 \pm 5.2$ & $67.8 \pm 5.5$ & 0.9747 \\
EDV $\left(\mathrm{ml} / \mathrm{m}^{2}\right)$ & $74.0 \pm 13.6$ & $69.7 \pm 11.9$ & 0.3013 \\
ESV $\left(\mathrm{ml} / \mathrm{m}^{2}\right)$ & $25.3 \pm 7.3$ & $22.6 \pm 5.7$ & 0.1957 \\
LV mass-ED $\left(\mathrm{g} / \mathrm{m}^{2}\right)$ & $82.5 \pm 17.5^{*}$ & $71.3 \pm 13.6^{*}$ & $0.0316^{*}$ \\
LV trab-ED $\left(\mathrm{g} / \mathrm{m}^{2}\right)$ & $25.0 \pm 6.6^{*}$ & $19.4 \pm 2.6^{*}$ & $0.0016^{*}$ \\
\hline
\end{tabular}

The parameters are converted to body surface area

$E D V$ end-diastolic volume, $E F$ ejection fraction, $E S V$ end-systolic volume, $L V$ mass-ED left ventricular end-diastolic myocardial mass, $L V$ trab-ED left ventricular end-diastolic papillary and trabecular mass, $N C-C M P$ noncompaction cardiomyopathy

$* \mathrm{p}<0.05$ 
declaration and its later amendments or comparable ethical standards. Ethical approval was obtained from the Central Ethics Committee of Hungary, and all participants provided informed consent.

\section{Image acquisition and study protocol}

CMR imaging was performed on the participants within 1 year using a 1.5 T MR scanner (Achieva, Philips Medical Systems, Eindhoven, The Netherlands) and a 5-channel cardiac coil. Retrospectively gated, balanced steady-state free precession (bSSFP) cine images were acquired in conventional 2-, 3-, and 4-chamber long-axis views. Breathhold SA cine images from base to apex were obtained. The slice thickness was $8 \mathrm{~mm}$ with no gap. After the SA cine images were acquired, either gadobutrol ((GA), Gadovist, Bayer-Schering, $0.16 \mathrm{ml} / \mathrm{kg}$ ) or gadobenate dimenglumine ((GD), MultiHance, Bracco, $0.25 \mathrm{ml} / \mathrm{kg}$ ) was injected intravenously. Each included individual received only one type of $\mathrm{CA}$, which was decided randomly. GA was administered to $12 \mathrm{NC}-\mathrm{CMP}$ patients and 12 healthy normal participants, and GD was administered to 8 NC-CMP patients and 7 healthy participants (Table 1). After the contrast material was injected, another set of SA cine images was started after 2 min in the same location (Fig. 2).

\section{Image analysis}

Semiautomatic tracing with manual correction of the epicardial borders was performed on SA images and was corrected by one medical professional (A. Sz., 7 years of experience) with excellent intraobserver agreement. The global concordance correlation coefficient, which represents the intraobserver agreement of all measured left ventricular parameters, was 0.88 (interpreted as: greater than 0.75 excellent).

We used threshold-based PTM quantification analytical software (the MassK module of 7.6 QMass Medis, Leiden, The Netherlands) for quantitative image analysis.

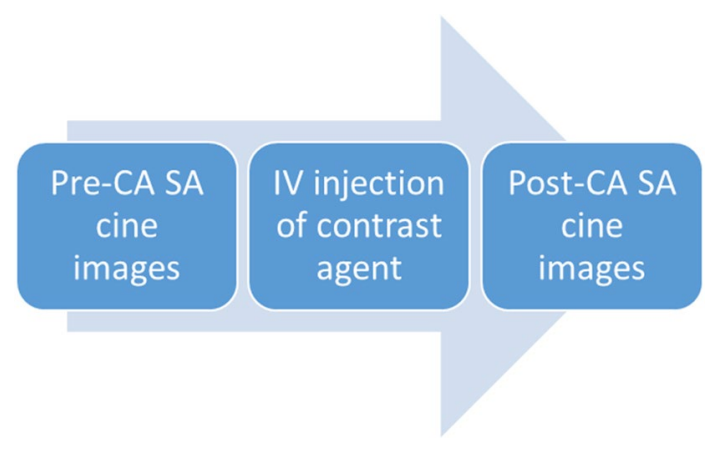

Fig. 2 Study protocol. Pre-CA: pre-contrast agent, post-CA: postcontrast agent, SA: short axis
This algorithm calculates the blood percentage value of each pixel based on the differing signal intensities of the blood pool and myocardial tissue [2, 3]. The signal intensity threshold was set to the default (50\%) (Fig. 3). Both the first (before CA administration (pre-CA)) and the second (after CA administration (post-CA)) SA scans were analyzed.

The following left ventricular parameters were calculated and converted to body surface area: end-systolic volume (ESV), end-diastolic volume (EDV), ejection fraction (EF), left ventricular end-diastolic myocardial mass (LVmass-ED), and left ventricular end-diastolic papillary and trabecular mass (LVtrab-ED). The normal left ventricular dimensions provided by Alfakih et al. were used as reference data which were established without the administration of contrast agent [12].

\section{Statistical analysis}

The Shapiro-Wilk test was applied to assess the normality of the distribution of the data. All data are described as the mean and standard deviation. A paired-sample t-test was used to assess differences in parameters that had a normal distribution; otherwise, the Wilcoxon rank sum test was used. p values less than 0.05 were considered significant. MedCalc Statistical Software version 17.9.5 (MedCalc Software, Ostend, Belgium) was used for statistical calculations.

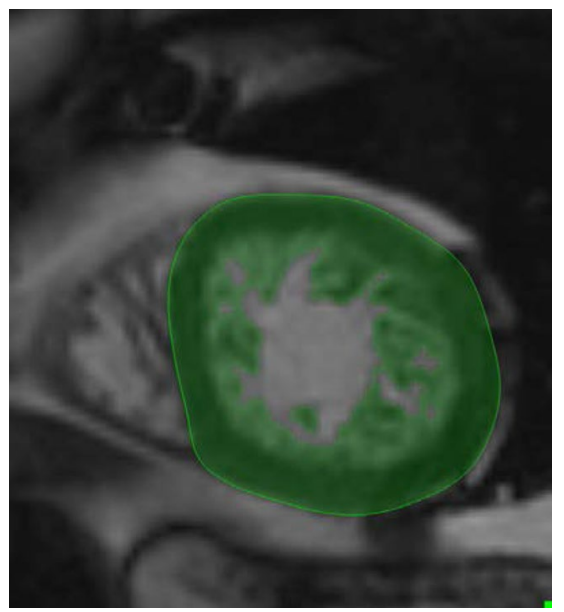

Fig. 3 Image analysis with the threshold-based papillary and trabeculated muscle quantification software. The green area represents the myocardial mass including the endocardial trabeculation of the left ventricle 


\section{Results}

\section{Comparison of the pre- and post-CA scans}

We compared the parameters calculated from the pre$\mathrm{CA}$ and post-CA scans both in the NC-CMP and healthy groups and found significant differences in the left ventricular parameters; the EDV and ESV were significantly larger, and the left ventricular myocardial mass and trabecular mass were significantly smaller on the post-CA scans in both groups (Table 2). Next, the values of the post-CA parameters were subtracted from the values of the pre-CA parameters, and the absolute values were used to compare the differences between the pre- and post-CA parameters of the two groups; the difference between the scans was significantly larger in the NC-CMP group than in the healthy normal group (NC-CMP difference vs. healthy normal difference: $\mathrm{EDV}, 7.9 \pm 6.0$ vs. $3.3 \pm 3.3 \mathrm{ml} / \mathrm{m}^{2}$; ESV, $5.4 \pm 6.3$ vs. $2.0 \pm 2.1 \mathrm{ml} / \mathrm{m}^{2}$; EF, $3.3 \pm 2.7$ vs. $2.3 \pm 2.7 \%$; LVmassED, $7.0 \pm 4.8$ vs. $3.3 \pm 2.1 \mathrm{~g} / \mathrm{m}^{2} ;$ LVtrab-ED, $6.0 \pm 3.9$ vs. $\left.3.2 \pm 1.7 \mathrm{~g} / \mathrm{m}^{2} ; \mathrm{p}<0.005\right)$.

\section{Comparing the effects of different CAs}

Since different types of CAs are in use, we tested whether these agents have similar effects on the studied parameters. We first compared the pre- and post-CA results in the NCCMP group (pre-GA vs. post-GA and pre-GD vs. post-GD). We found results similar to those obtained in the comparison of the total pre-CA versus post-CA scans. Regardless of the applied contrast material, the EDV, ESV and EF values were significantly larger, while the LVmass-ED and LVtrab-ED values were significantly smaller when calculated from the post-CA scans (Table 3). We performed these comparisons in the healthy normal group as well and obtained similar results; the LVtrab-ED values were significantly lower in the post-CA scans for both contrast materials. LVmass-ED values were also significantly lower in the post-GA scans, and EDV values were significantly higher in the post-GD scans (Table 3).

\section{Comparison of the CAs to each other}

Finally, we compared the GA and GD CAs to each other in the healthy and NC-CMP groups (pre-GA vs. pre-GD). Because the parameters calculated from the pre-CA scans did not differ significantly between the healthy and patient groups, we compared the post-CA scans and found no significant difference between the groups (Table 4).

\section{Discussion}

CMR imaging is currently the gold standard for measuring cardiac volume and function and myocardial mass [13-16]. In recent years, an increasing number of postprocessing programs have been equipped with threshold-based papillary and trabeculated muscle-quantifying algorithms, leading to easier and faster evaluation and more accurate cardiac volumes and masses [2,3].

This study was designed to confirm our experience with the postprocessing evaluation of scans made after the injection of gadolinium-based contrast material, namely, that endocardial trabeculation is harder to detect. We studied the effect of CAs on the applicability of threshold-based PTM quantification software in patients with left ventricular noncompaction and in healthy normal study subjects.

Our results showed that the EDV and ESV values calculated from post-CA scans were significantly higher, while the ED-mass and LVtrab-ED values calculated from postCA scans were significantly lower than those calculated from pre-CA images in the NC-CMP and healthy normal groups. However, the difference between the pre-CA and post-CA parameters was significantly larger in the patient group than in the healthy group.
Table 2 Calculated parameters of the noncompaction cardiomyopathy and healthy study groups calculated from pre-contrast and post-contrast scans

\begin{tabular}{|c|c|c|c|c|c|c|}
\hline \multicolumn{4}{|l|}{ NC-CMP } & \multicolumn{3}{|l|}{ Healthy } \\
\hline & Pre-CA & Post-CA & $\mathrm{p}$ & Pre-CA & Post-CA & $\mathrm{p}$ \\
\hline $\operatorname{ESV}\left(\mathrm{ml} / \mathrm{m}^{2}\right)$ & $25.3 \pm 7.3$ & $30.1 \pm 11.2$ & $0.0059^{*}$ & $22.6 \pm 5.7$ & $23.9 \pm 6.3$ & $0.0411 *$ \\
\hline $\operatorname{EDV}\left(\mathrm{ml} / \mathrm{m}^{2}\right)$ & $74.0 \pm 13.6$ & $81.1 \pm 16.3$ & $0.0002^{*}$ & $69.7 \pm 11.9$ & $72.2 \pm 10.7$ & $0.0121 *$ \\
\hline $\mathrm{EF}(\%)$ & $66.1 \pm 5.2$ & $64.7 \pm 5.8$ & 0.1608 & $67.8 \pm 5.5$ & $67.1 \pm 6.4$ & 0.7086 \\
\hline LV mass-ED $\left(\mathrm{g} / \mathrm{m}^{2}\right)$ & $82.5 \pm 17.5$ & $75.7 \pm 15.9$ & $<0.0001 *$ & $71.3 \pm 13.6$ & $68.7 \pm 13.9$ & $0.0010^{*}$ \\
\hline LV trab-ED $\left(\mathrm{g} / \mathrm{m}^{2}\right)$ & $25.0 \pm 6.6$ & $18.9 \pm 4.7$ & $<0.0001 *$ & $19.4 \pm 2.6$ & $16.2 \pm 3.0$ & $<0.0001 *$ \\
\hline
\end{tabular}

The parameters are converted to body surface area

$E D V$ end-diastolic volume, $E F$ ejection fraction, $E S V$ end-systolic volume, $L V$ mass-ED left ventricular end-diastolic myocardial mass, $L V$ trab-ED left ventricular end-diastolic papillary and trabecular mass, $N C$-CMP noncompaction cardiomyopathy, post-CA post- contrast agent, pre-CA pre- contrast agent $* \mathrm{p}<0.05$ 
Table 3 Comparing the effect of gadobutrol (GA) and gadobenate dimenglumine (GD) on the calculated parameters
Table 4 Comparison of the gadobutrol (GA) and gedobenate dimenglumine (GD) receiving populations' pre-and post-contrast scans

\begin{tabular}{lllllll}
\hline & Pre-GA & Post-GA & $\mathrm{p}$ & Pre-GD & Post-GD & $\mathrm{p}$ \\
\hline NC-CMP & & & & & & \\
ESV $\left(\mathrm{ml} / \mathrm{m}^{2}\right)$ & $24.1 \pm 7.2$ & $28.9 \pm 12.6$ & $0.0269^{*}$ & $27.2 \pm 7.6$ & $31.8 \pm 9.0$ & $0.0013^{*}$ \\
EDV $\left(\mathrm{ml} / \mathrm{m}^{2}\right)$ & $71.7 \pm 12.7$ & $79.5 \pm 17.8$ & $0.0076^{*}$ & $77.4 \pm 15.0$ & $83.6 \pm 14.6$ & $0.0046^{*}$ \\
EF $(\%)$ & $66.6 \pm 5.8$ & $66.3 \pm 5.3$ & 0.8025 & $65.3 \pm 4.4$ & $62.4 \pm 6.0$ & $0.0425^{*}$ \\
LV mass-ED $\left(\mathrm{g} / \mathrm{m}^{2}\right)$ & $81.4 \pm 19.6$ & $75.6 \pm 17.4$ & $0.0031^{*}$ & $84.3 \pm 15.0$ & $75.9 \pm 14.5$ & $0.0008^{*}$ \\
LV trab-ED $\left(\mathrm{g} / \mathrm{m}^{2}\right)$ & $24.7 \pm 7.8$ & $18.8 \pm 5.1$ & $0.0014^{*}$ & $25.4 \pm 4.9$ & $19.1 \pm 4.4$ & $0.0001^{*}$ \\
Healthy & & & & & & \\
ESV $\left(\mathrm{ml} / \mathrm{m}^{2}\right)$ & $22.5 \pm 5.0$ & $23.9 \pm 6.1$ & 0.0771 & $22.7 \pm 7.1$ & $23.9 \pm 7.1$ & 0.3054 \\
EDV $\left(\mathrm{ml} / \mathrm{m}^{2}\right)$ & $69.2 \pm 9.5$ & $71.2 \pm 7.2$ & 0.1627 & $70.5 \pm 16.2$ & $73.9 \pm 15.6$ & $0.0075^{*}$ \\
EF $(\%)$ & $67.5 \pm 5.9$ & $66.7 \pm 6.9$ & 0.7334 & $68.2 \pm 5.1$ & $67.9 \pm 5.7$ & 0.8034 \\
LV mass-ED $\left(\mathrm{g} / \mathrm{m}^{2}\right)$ & $71.3 \pm 12.3$ & $68.4 \pm 12.8$ & $0.0065^{*}$ & $71.3 \pm 16.6$ & $69.1 \pm 16.5$ & 0.0993 \\
LV trab-ED $\left(\mathrm{g} / \mathrm{m}^{2}\right)$ & $19.7 \pm 2.2$ & $16.4 \pm 3.0$ & $0.0001^{*}$ & $19.0 \pm 3.4$ & $15.9 \pm 3.4$ & $0.0015^{*}$ \\
\hline
\end{tabular}

The parameters are converted to body surface area

$E D V$ end-diastolic volume, $E F$ ejection fraction, $E S V$ end-systolic volume, $L V$ mass-ED left ventricular end-diastolic myocardial mass, LVtrab-ED left ventricular end-diastolic papillary and trabecular mass, $N C$-CMP non-compaction cardiomyopathy, post-GA post-gadobutrol, post-GD post- gadobenate gimenglumine, pre-GA pre-gadobutrol, pre-GD pre-gadobenate dimenglumine $* \mathrm{p}<0.05$

\begin{tabular}{|c|c|c|c|c|c|c|}
\hline & \multicolumn{3}{|l|}{ Pre-CA } & \multicolumn{3}{|l|}{ Post-CA } \\
\hline & GA & GD & $\mathrm{p}$ & GA & GD & $\mathrm{p}$ \\
\hline \multicolumn{7}{|l|}{ NC-CMP } \\
\hline $\operatorname{ESV}\left(\mathrm{ml} / \mathrm{m}^{2}\right)$ & $42.3 \pm 10.2$ & $46.7 \pm 8.1$ & 0.9453 & $44.1 \pm 10.8$ & $49.6 \pm 9.1$ & 0.8438 \\
\hline $\operatorname{EDV}\left(\mathrm{ml} / \mathrm{m}^{2}\right)$ & $73.4 \pm 11.3$ & $77.4 \pm 15.0$ & 0.5701 & $81.5 \pm 18.9$ & $83.6 \pm 14.6$ & 0.8299 \\
\hline $\mathrm{EF}(\%)$ & $65.4 \pm 6.3$ & $65.3 \pm 4.4$ & 0.9484 & $64.4 \pm 5.1$ & $62.4 \pm 6.0$ & 0.5706 \\
\hline LV mass-ED $\left(\mathrm{g} / \mathrm{m}^{2}\right)$ & $82.8 \pm 17.2$ & $84.3 \pm 15.0$ & 0.8513 & $77.0 \pm 14.0$ & $75.9 \pm 14.5$ & 0.8899 \\
\hline LV trab-ED $\left(\mathrm{g} / \mathrm{m}^{2}\right)$ & $24.0 \pm 6.3$ & $25.4 \pm 4.9$ & 0.8919 & $19.4 \pm 4.6$ & $19.1 \pm 4.4$ & 0.8919 \\
\hline \multicolumn{7}{|l|}{ Healthy } \\
\hline $\operatorname{ESV}\left(\mathrm{ml} / \mathrm{m}^{2}\right)$ & $24.6 \pm 3.8$ & $22.7 \pm 7.1$ & 0.5245 & $25.5 \pm 4.5$ & $23.9 \pm 7.1$ & 0.6847 \\
\hline $\operatorname{EDV}\left(\mathrm{ml} / \mathrm{m}^{2}\right)$ & $72.1 \pm 9.1$ & $70.5 \pm 16.2$ & 0.8346 & $72.8 \pm 8.1$ & $73.9 \pm 15.6$ & 0.8715 \\
\hline $\mathrm{EF}(\%)$ & $65.6 \pm 5.1$ & $68.2 \pm 5.1$ & 0.4415 & $65.0 \pm 4.5$ & $67.9 \pm 5.7$ & 0.4459 \\
\hline LV mass-ED $\left(\mathrm{g} / \mathrm{m}^{2}\right)$ & $72.5 \pm 12.1$ & $71.3 \pm 16.6$ & 0.8756 & $72.8 \pm 8.1$ & $73.9 \pm 15.6$ & 0.8715 \\
\hline LV trab-ED $\left(\mathrm{g} / \mathrm{m}^{2}\right)$ & $19.7 \pm 2.2$ & $19.0 \pm 3.4$ & 0.6321 & $16.0 \pm 2.6$ & $15.9 \pm 3.4$ & 0.9451 \\
\hline
\end{tabular}

The parameters are converted to body surface area

$E D V$ end-diastolic volume, $E F$ ejection fraction, $E S V$ end-systolic volume, $L V$ mass-ED left ventricular end-diastolic myocardial mass, LVtrab-ED left ventricular end-diastolic papillary and trabecular mass, $N C$-CMP non-compaction cardiomyopathy, post-GA post-gadobutrol, post-GD post- gadobenate gimenglumine, pre-GA pre-gadobutrol, pre-GD pre-gadobenate dimenglumine

$* \mathrm{p}<0.05$

The signal intensity of SSFP images depends on the T2/ T1 ratio of the tissue of interest. Gadolinium-based extracellular CAs decrease the T1 values of blood and myocardial tissue, which results in increased signal intensity on SSFP images. This effect is more pronounced in the myocardium and less pronounced in the blood pool, leading to decreased contrast between the two tissues. T2 values are slightly reduced by CAs administered at low doses $(0.1-0.3 \mathrm{mmol} /$ $\mathrm{kg}$ ), and these changes are overridden by $\mathrm{T} 1$ shortening; thus, changes in signal intensity after administration of a CA are due to changes in T1 values [17-19]. The end result of these changes in relaxivity is that the difference between the $\mathrm{T} 1 / \mathrm{T} 2$ ratios of the blood and the myocardium decreases after contrast administration. As the mechanism of threshold-based quantification is based on the high signal intensity difference between the blood and the myocardium, our results suggest that this effect has a significant impact on the detection of endocardial trabeculae on postcontrast scans, 
not only in patients with left ventricular hypertrabeculation but also in patients with normal trabeculation. Our results correlate with those from a study about the precision of another technique (feature tracking) from a different vendor on post-CA scans; in that study, a contrast agent significantly changed the measured strain values, which also confirmed the importance of the signal intensity-altering effect of contrast agents [11]. These results are important both during regular CMR postprocessing and in research projects for standardizing protocols.

We also studied the effect of two different contrast materials on the precision of threshold-based software to determine whether the change in the calculated parameters depends on the type of CA applied. The LVtrab-ED value was significantly smaller and the EDV value was larger on both postGA and post-GD images. However, no significant differences were found in the comparison between the two CAs.

Gadolinium is a paramagnetic extracellular CA that shortens the $\mathrm{T} 1$ and $\mathrm{T} 2$ relaxation time of the surrounding protons, which increases signal intensity on $\mathrm{T} 1$-weighted images [20]. Different chelators are used to create complexes with gadolinium; therefore, different products are available. Compared to traditional extracellular gadolinium CAs, GD binds weakly to albumin and in this way attenuates the signal intensity of blood, has a slight intravascular effect and prolongs the plasma half-life. GA does not bind to proteins but reduces T1 values more than GD because of its concentration [21-23]. These properties do not seem to make a significant difference regarding the effect studied here.

In summary, this study reveals that the threshold-based PTM-quantifying software provides altered results when SA scans are made after the injection of contrast material, as the signal intensity difference between the blood and the myocardium is decreased on these postcontrast images. This effect is independent of the type of CA applied and the amount of endocardial trabeculation. In this study, CAs influenced the measured values, especially in patients with excessive endocardial trabeculation; therefore, the method of evaluation should be standardized. Further studies are required to evaluate this phenomenon with myocardial hypertrabeculation.

\section{Limitations}

The main limitation is the small number of included patients, as noncompaction cardiomyopathy is a relatively rare disease. Furthermore, some patients did not consent to receive the CA; thus, we could not include those patients.

Acknowledgments Open access funding provided by Semmelweis University (SE). The study was supported by National Research,
Development and Innovation Office (NKFIH) of Hungary (Grant No. NVKP_16-1-2016-0017).

Funding The study was supported by the National Research, Development and Innovation Office (NKFIH) of Hungary (Grant No. NVKP_16-1-2016-0017).

\section{Compliance with ethical standards}

Conflict of interest We have no potential conflict of interest.

Open Access This article is distributed under the terms of the Creative Commons Attribution 4.0 International License (http://creativeco mmons.org/licenses/by/4.0/), which permits unrestricted use, distribution, and reproduction in any medium, provided you give appropriate credit to the original author(s) and the source, provide a link to the Creative Commons license, and indicate if changes were made.

\section{References}

1. Petitjean C, Dacher JN (2011) A review of segmentation methods in short axis cardiac MR images. Med Image Anal 15(2):169-184

2. Varga-Szemes A et al (2016) Clinical feasibility of a myocardial signal intensity threshold-based semi-automated cardiac magnetic resonance segmentation method. Eur Radiol 26(5):1503-1511

3. Jaspers K et al (2013) Improving the reproducibility of MR-derived left ventricular volume and function measurements with a semi-automatic threshold-based segmentation algorithm 29(3):617-623

4. Freling HG et al (2013) Impact of right ventricular endocardial trabeculae on volumes and function assessed by CMR in patients with tetralogy of Fallot. Int J Cardiovasc Image 29(3):625-631

5. Janik M et al (2008) Effects of papillary muscles and trabeculae on left ventricular quantification: increased impact of methodological variability in patients with left ventricular hypertrophy. J Hypertens 26(8):1677-1685

6. Csecs I et al (2018) Left and right ventricular parameters corrected with threshold-based quantification method in a normal cohort analyzed by three independent observers with various training-degree. Int J Cardiovasc Imaging 34:1127-1133

7. Almeida AG, Pinto FJ (2014) Republished: non-compaction cardiomyopathy. Postgrad Med J 90(1062):208

8. Jain A et al (2008) Role of cardiovascular magnetic resonance imaging in arrhythmogenic right ventricular dysplasia. J Cardiovasc Magn Reson 10:32

9. Budjan J et al (2016) Rapid functional cardiac imaging after gadolinium injection: evaluation of a highly accelerated sequence with sparse data sampling and iterative reconstruction. Sci Rep 6:38236

10. D'Angelo T et al (2017) High-throughput gadobutrol-enhanced CMR: a time and dose optimization study. J Cardiovasc Magn Reson 19(1):83

11. Kuetting DL et al (2016) The effects of extracellular contrast agent (Gadobutrol) on the precision and reproducibility of cardiovascular magnetic resonance feature tracking. J Cardiovasc Magn Reson 18(1):30

12. Alfakih K et al (2003) Normal human left and right ventricular dimensions for MRI as assessed by turbo gradient echo and steady-state free precession imaging sequences. J Magn Reson Imaging 17(3):323-329 
13. Katz J et al (1988) Estimation of human myocardial mass with MR imaging. Radiology 169(2):495-498

14. Sechtem U et al (1987) Measurement of right and left ventricular volumes in healthy individuals with cine MR imaging. Radiology 163(3):697-702

15. Grothues F et al (2004) Interstudy reproducibility of right ventricular volumes, function, and mass with cardiovascular magnetic resonance. Am Heart J 147(2):218-223

16. Semelka RC et al (1990) Normal left ventricular dimensions and function: interstudy reproducibility of measurements with cine MR imaging. Radiology 174(3 Pt 1):763-768

17. Krombach GA et al (2011) Functional cardiac MR imaging with true fast imaging with steady-state free precession before and after intravenous injection of contrast medium: comparison of image quality and accuracy. Eur Radiol 21(4):702-711

18. Pennell DJ, Underwood SR, Longmore DB (1993) Improved cine MR imaging of left ventricular wall motion with gadopentetate dimeglumine. J Magn Reson Imaging 3(1):13-19

19. Kirsch JE (1991) Basic principles of magnetic resonance contrast agents. Top Magn Reson Imaging 3(2):1-18
20. Xiao YD et al (2016) MRI contrast agents: classification and application (review). Int J Mol Med 38(5):1319-1326

21. Scott L (2013) Gadobutrol: a review of its use for contrastenhanced magnetic resonance imaging in adults and children. Clin Drug Investig 33(4):303-314

22. Seale MK et al (2009) Hepatobiliary-specific MR contrast agents: role in imaging the liver and biliary tree. Radiographics 29(6): 1725-1748

23. Wildgruber $\mathrm{M}$ et al (2014) Single-dose gadobutrol in comparison with single-dose gadobenate dimeglumine for magnetic resonance imaging of chronic myocardial infarction at $3 \mathrm{~T}$. Invest Radiol 49(11):728-734

Publisher's Note Springer Nature remains neutral with regard to jurisdictional claims in published maps and institutional affiliations. 